\title{
FAST CONVERSION FACTOR (GAIN) MEASUREMENT OF A CCD USING IMAGES WITH VERTICAL GRADIENT
}

\author{
Fabrice Christen ${ }^{1}$, Konrad Kuijken ${ }^{1,2}$, Dietrich Baade $^{3}$, Cyril Cavadore ${ }^{4}$, \\ Sebastian Deiries ${ }^{3}$, Olaf Iwert ${ }^{3}$. \\ ${ }^{1}$ Kapteyn Astronomical Institute, Groningen, The Netherlands; ${ }^{2}$ Sterrewacht Leiden, Leiden, \\ The Netherlands; ${ }^{3}$ European Southern Observatory, Garching, Germany; ${ }^{4}$ Thales, France
}

Abstract: This paper describes a fast technique for estimating the Conversion Factor of a CCD. It is based on the adjustment of the standard photon transfer curve technique to 2 TDI (Time Delay Integration) images. The data used to test the procedure are recorded with the ESO test bench, in the context of characterizing the OmegaCAM CCDs. No modifications of the test bench are needed to use this technique. For five CCDs, results from the standard photon transfer method (also called variance method) and with the technique developed in this paper were compared. Results are compliant. This technique even if it is still under development with possible future improvements, proved reliable. It can be used for simple and efficient gain measurements of CCDs.

Key words: CCD Characterization, Conversion Factor, gain.

\section{INTRODUCTION}

During the tests of the OmegaCAM ${ }^{1}$ CCDs (Christen 2002), TDI images have been acquired using the ESO test bench (Amico 1996) to check the linearity of the chips. This paper will describe how to use these data to measure the conversion factor. This parameter reported in electrons per analog digital unit (ADU), relates the pixel value to the number of electrons

1 OmegaCAM is a one square degree wide field imager which will be mounted on the VST telescope in Paranal, Chile (Kuijken 2004, Iwert 2005). 
and will be extracted from the photon transfer curve derived from two TDI images.

After a description of the procedure in Section 2, the first results will be presented in Section 3. The conclusions are reported in section 4.

\section{PHOTON TRANSFER CURVE FROM TWO TDI IMAGES.}

This section will describe how TDI images ${ }^{2}$ can be used to measure the conversion factor.

To acquire two TDI images, the test bench is set as if flats have to be recorded. There is one particular condition; the test bench has to be able to open the shutter during the reading of the CCD. The monochromators are set to a wavelength of $630 \mathrm{~nm}$ and $10 \mathrm{~nm}$ bandwidth. In this condition, $44-82 \mathrm{e} 2 \mathrm{v}$ CCDs photon response non uniformities are negligible ( 1\%). Most of the noise is photon noise. Equally important, the illumination of the CCD has to be homogeneous all across the surface (the ESO test bench achieved this criteria. it produces a flat illumination with fluctuations which are less than $1 \%$ ). Under these conditions, it is not necessary to flat field out the TDI images. Otherwise, the method described in this paper is still usable, but it would involve more computation process stages.

The principle and the mathematical tools are the same as in the standard photon transfer curve technique (Janesick 2001). The variance in Analog

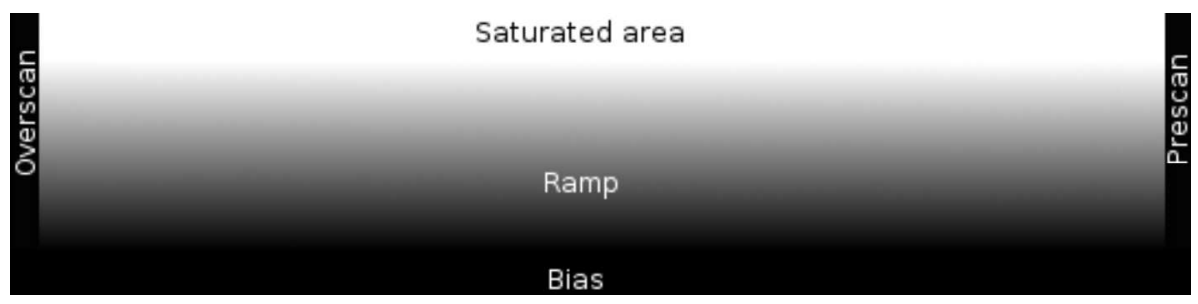

Figure 1. Example of a TDI image recorded using the ESO test bench. Here is a 2148x512 pixels size image. A legend has been added. The area where the ramp shows up is also called in this paper "sensitive area".

2 A TDI acquisition consists in collecting light and reading out continuously the detector one row of pixels at a time from the bottom (where the output register is located) of a detector chip. Under a constant illumination, this reading out mode will create an image (see Fig. 1) with the required response. 


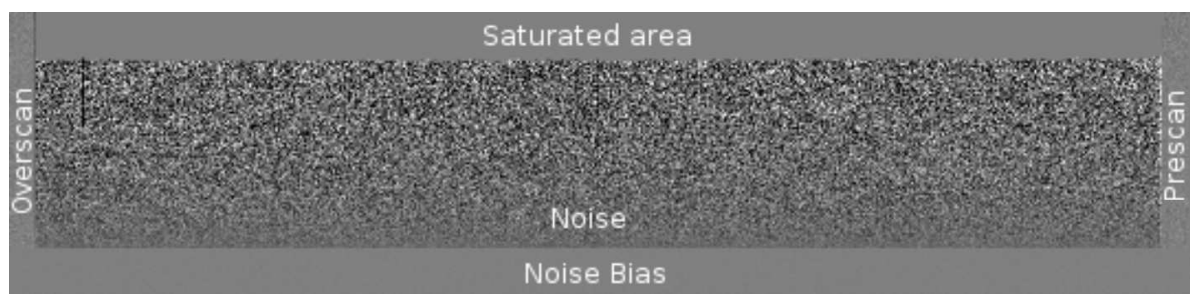

Figure 2. Example of noise pattern after subtraction of two TDI images.

Digital Units is related to the signal (ADU) by the following linear equation,

$$
\sigma_{a}^{2}=\frac{1}{g} \overline{S_{a}}+\frac{1}{g^{2}} \sigma_{r o n}^{2}
$$

$g \quad:$ Conversion factor (e-/ADU)

$S_{a}:$ Average pixel value (ADU)

$\sigma_{a}:$ Total rms noise (ADU)

$\sigma_{r o n}:$ read out rms noise (e)

The variance of the signal will be plotted versus the mean signal. To extract these parameters the following manipulations have to be carried out.

To measure the mean signal, the two TDI images are averaged. In the sensitive area, the mean of each row, $\overline{S_{a}}$, is measured and the bias subtracted.

To measure the variance one TDI image is subtracted from the other (see Fig. 2). For each row of the sensitive area, the variance, $\sigma^{2}$, is measured $\left(\sigma_{a}^{2}=\sigma^{2} / 2\right.$. The variance is divided by two to obtain the variance of the line in a single frame).

The plot of the $\left(\overline{S_{a}}, \sigma_{a}^{2}\right)$ pairs will provide the photon transfer curve and the linear fit of this plot, the slope which is the inverse of the conversion factor.

\section{RESULTS}

To test the gain measurement with two TDI images, sets of data from five CCDs are used. Based on the procedure developed above, the photon transfer curve for each CCD is plotted (see for example Fig. 3) and the conversion factor calculated. The results are in Table I. The gain measured with one (See Appendix B) and two TDI images are in accordance with the 


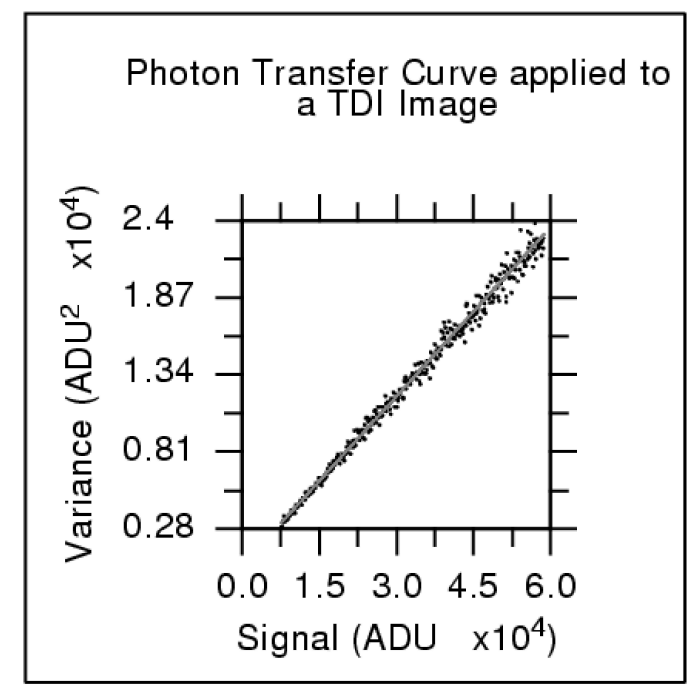

Figure 3. Example of a photon transfer curve realized with two TDI images.

Variance Method (See Appendix A) used in the OmegaCAM test procedure. The maximum difference in this set is $1.5 \%$. These results are very promising.

The Variance Method used to measure the gain in the OmegaCAM procedure has been compared to the measurements of the gain made with the ${ }^{55} \mathrm{Fe}$ method. The results show that both techniques are in accordance.

Further confidence to the method is related to non linearity. When this defect is observed in the standard photon curve, the photon curve from TDI images shows the defect too.

Table I. Gain measured for five CCDs with three different methods.

\begin{tabular}{cccc}
\hline CCD serial number & \multicolumn{3}{c}{ Gain (e-/ADU) obtained from } \\
& One TDI Image & Two TDI Images & Variance method \\
\hline $02263-07-02$ & $2.60 \pm 0.01$ & $2.58 \pm 0.01$ & $2.59 \pm 0.02$ \\
$02111-01-02$ & $2.51 \pm 0.01$ & $2.50 \pm 0.01$ & $2.52 \pm 0.02$ \\
$00152-01-02$ & $2.64 \pm 0.01$ & $2.62 \pm 0.01$ & $2.58 \pm 0.01$ \\
$02111-05-02$ & $2.50 \pm 0.01$ & $2.50 \pm 0.01$ & $2.51 \pm 0.01$ \\
$02111-13-01$ & $2.62 \pm 0.01$ & $2.60 \pm 0.01$ & $2.62 \pm 0.01$ \\
\hline
\end{tabular}

In column one, the serial number of the CCDs, in column two, the gain measured with one TDI image, in column three, the gain from two TDI images and in column four, the gain measured with the photon transfer technique. 


\section{CONCLUSION}

This paper has described a fast technique to estimate the Conversion Factor (Gain) of the CCD. It is based on the photon transfer curve adjusted to two TDI images. It has been tested on data taken at ESO Garching by the Optical Detector Team (ODT) team to characterize the OmegaCAM CCDs. The TDI images are taken during the ESO CCD test procedure to measure the linearity of the chips. The preliminary results to measure the gain with these data are very promising. They are in agreement with the standard gain measurement method.

This technique has the advantage to use the ESO Test bench without any modification, provides much more points for the photon transfer curve (here in our test about 400 points per plot compare to 20 usually) and is very quick. Only two TDI images are required to measure the Conversion Factor. If a set of flat fields is considered to correct the photon response non uniformity, the linearity and residual-non-linearity can also be calculated (Cavadore 1999, private communication).

Due to the fact that the gain measurement with this method is faster than the standard one, it turns out to be appropriate to use it during the tuning of CCDs (i.e. voltage adjustment). This technique even if further work is needed to improve it, already proved reliable and can be used for simple and efficient gain measurements of CCDs.

\section{ACKNOWLEDGMENTS}

Fabrice Christen would like to thank all the members of the Optical Detector Team for their help during the tests of the OmegaCAM CCDs and without whom he would not have won the award of the 'Young Achiever' during the 2005 Scientific Detector Workshop.

Fabrice Christen thanks also OmegaCEN data center for providing its help and facilities.

\section{APPENDIX A, THE VARIANCE METHOD}

The standard method used to measure the conversion factor with the ESO test bench is based on the photon transfer method (or variance method). Two flat fields and two bias exposures are acquired. The bias frames are subtracted from the flat frames. These bias corrected images are divided, one by the other, and the result is multiplied by the mean value of one of the 
image. To get the final result, 100 sub-windows are selected and in each of them the mean signal and the variance are measured. Then the gains $G$ are measured. The median gain is calculated over all the sub windows. The gain's error is the standard deviation of the 100 conversion factors measured.

\section{APPENDIX B, PHOTON TRANSFER CURVE FROM ONE TDI IMAGE}

The major problem to measure the gain with TDI images is the precise repetition of data acquisition. It can be difficult to start the integration always at the same line index if the shutter suffers from random delay when opening. To get round to this problem, the photon transfer curve with one TDI image can be used (Janesick 2001, Chapter 2.2.10.2 "Shutterless Photon Transfer"). The mean and the variance of the ramp are calculated for each line and the pair of points (intensity, variance) plotted.

In normal circumstance, in a flat field for example, the variance measured will not provide you the shot noise but instead, the shot noise plus the pixel to pixel non uniformity variation.

Here, in the case of a TDI image, the quantity of electrons in a pixel is the sum of all the elementary flux it received during its complete transfer until the output register. Except in the first few lines read out, the pixel intensity in the output image is thus barely affected by the pixel to pixel non uniformity.

That's why in this paper, with this technique which uses only one TDI image, the twenty first rows (minimum) of the sensitive area have to be discarded to measure the gain. In these first lines, pixel to pixel non uniformities bias the measurements of the variance, and, because of the propagation of the errors, the estimation of the gain.

References

Amico P. and Böhm T., 1996, Optical Detectors for Astronomy, Kluwer Academic Publishers, Dordrecht, Holland, pp. 95-105.

Christen F. et al, 2002, Scientific Detectors for Astronomy, The beginning of a New Era, Kluwer Academic Publishers, Dordrecht, Holland, pp. 485-488.

Iwert O., 2005, these proceedings, "The OmegaCAM 16k x 16k CCD Detector System for the ESO VLT", Spring.

Janesick J. R., 2001, Scientific Charge-Coupled Devices, SPIE press monograph, Bellingham Washington.

Kuijken K. et al, 2004, Proceedings of the SPIE, Volume 5492, pp. 484-493. 